\title{
Attributes, Attitudes, and Motivations of Personnel Involved with Sports Physical Therapy Residency Training
}

\author{
Edward P Mulligan, PT, DPT, SCS, OCS, ATC ${ }^{1}$ (i) $^{\text {a }}$ \\ 1 Physical Therapy, UT Southwestern School of Health Professions \\ Keywords: sports physical therapy, residency, post-professional training, motivations, fellowship \\ https://doi.org/10.26603/001c.25465
}

\section{International Journal of Sports Physical Therapy}

Vol. 16, Issue 4, 2021

\section{Background}

Post-professional residency training in sports physical therapy has undergone rapid growth since its inception over 20 years ago with 58 programs currently accredited.

\section{Purpose}

The purpose of this survey was to describe and contrast the demographics, motivations, and selection influences from the perspective of both potential training applicants and program faculty.

\section{Study Design}

Cross-sectional descriptive survey

\section{Methods}

156 physical therapists identified as stakeholders in sports residency and fellowship training were invited to participate in a 115-item survey. Descriptive measures of central tendencies to describe the data and Mann Whitney Rank Sum tests were used to detect differences between the perspectives of applicants and faculty.

\section{Results}

50 program faculty and 57 applicants responded to the survey for a $69 \%$ response rate. Motivations for post-professional training categorized as extremely important were largely intrinsic behavioral modifiers centering on improved knowledge, skills, and outcomes while satisfying a passion for sports specialty training and enhancing job opportunities in the field. 7 of the 10 highest rated application motivations were rated as significantly more important by applicants than faculty members $(p<0.05)$. The two most highly rated influences for choosing to apply to a specific residency site were the perception for subsequent job opportunities and perceived relationship and qualifications with the residency director and staff. The importance of job opportunities in sports PT was rated much higher by the applicant than the faculty $(p=0.003)$.

\section{Conclusions}

While the motivations for residency training may be slightly different between groups the importance of information acquisition and methods for residency selection criteria seem more congruent. Residency faculty may underestimate the importance of some of the most important motivations that prompt interest in residency training. Recognition of these factors may alter the presentation and content design of residency curriculums.

\footnotetext{
a Corresponding author: 


\section{INTRODUCTION}

Physical therapy residency and fellowship training in the United States is a recognized and promoted path to afford advanced training opportunities in specialty practice areas. The content of the specialty expertise is captured by the description of residency practice and governed by the American Board of Residency and Fellowship Education. ${ }^{1-7}$ As of January of 2021 there were 58 accredited sports physical therapy residency programs with an additional 14 programs in a developmental or candidacy status. ${ }^{8}$ Additionally, there are nine fellowship training programs relevant to the practice of sports physical therapy (performing arts, upper extremity athlete, and D1 athlete) and three more fellowship programs in the development stage. ${ }^{8}$ Residency and fellowship programs exist to improve skill and expertise, provide structured mentoring, expose the trainee to event coverage, and potentially offer an accelerated track to attaining clinical specialist recognition.

In part, the popularity and exponential growth in sports physical therapy residency and fellowship training over the past two decades may be attributable to the opportunity for young, less experienced clinicians to interact with, learn from, and network with like-minded advanced practitioners. What is less clear is what specifically motivates clinicians to pursue this optional, post-professional training in this specific field of physical therapy. ${ }^{9-11}$ Even though residents and fellows usually have a high work demand and are compensated at a lower rate, the number of qualified applicants for these training opportunities far exceed the number of positions available. ${ }^{8}$ Even more striking is that this strong interest exists despite increasing stress over educational debt and the recognized value of return on investment analysis in regards to debt to income ratios. ${ }^{12}$ Despite all these factors, interest in sports physical therapy residency training remains very high. In light of this, programs providing advanced sports physical training often have a deep field of applicants from which to make a candidate selection. It is unclear what motivations, attributes, and attitudes make a residency or fellowship application competitive. It would be advantegous to both the program and applicant to know what characteristics enhance the match between these two entities of interest. Ideally, the application process will maximize the likelihood of the optimal training opportunity being provided by the strengths of particular training program. ${ }^{11,13-17}$

Given the importance of mentorship and direct, collegial communication between the resident and program faculty mentor(s), this study aims to evaluate the characteristics, components, and elements of the residency experience that are important to ensure a good match for both parties. ${ }^{14,15}$ The purpose of this study was to describe and contrast the demographics, motivations, and selection influences from the perspective of both potential training applicants and program faculty. This purpose has four principal objectives. First, to better understand the factors that motivate the pursuit of sports residency training and contrast how important these factors are to resident applicants versus faculty providers. Second, to identify factors that influence the match (application to or acceptance of residents to a training program). Third, to identify the importance and pre- ferred methods to acquire, exchange, and disseminate information about the program between the applicant and provider. And finally, provide insight regarding the factors and criterion used to differentiate and select applicants for residency positions.

\section{METHODS}

An online cross-sectional survey was designed, further described below, to collect information regarding the motivations, attitudes, and attributes of individuals involved with sports physical therapy residency and fellowship education.

\section{PARTICIPANTS}

Two groups of individuals from a sample of convenience were invited via email during May of 2020. All physical therapists listed as residency or fellowship directors on the directory provided by the American Board of Physical Therapy Residency and Fellowship Education (ABPTRFE) website as well as all members of the American Academy of Sports Physical Therapy (AASPT) Specialization special interest group was included. ${ }^{8,13}$ Because Residency and Fellowship Physical Therapy Centralized Activation Service (RF-PTCAS) and the ABPTRFE are prohibited from disseminating personal contact information on residency applicants and graduates we asked these 156 individuals to assist with distributing the invitation link. They were asked to forward the email invitation to all current applicants and all past graduates. The goal was to obtain at least 50 responses from both resident applicant and faculty member categories. This would represent at least an average of two responses from each program accredited at the time of the survey. Based on a $95 \%$ confidence level, at least 105 responses (67\% response rate) were needed from known invited group members to bring the statistical random sampling margin of error to within $\pm 5 \%$.

\section{SURVEY ADMINISTRATION}

Study data were collected and managed using REDCap electronic data capture tools hosted at UT Southwestern Medical Center in Dallas, TX. REDCap (Research Electronic Data Capture) is a secure, web-based application designed to support data capture for research studies, providing: 1) an intuitive interface for validated data entry, 2) audit trails for tracking data manipulation and export procedures, 3) automated export procedures for seamless data downloads to common statistical packages, and 4) procedures for importing data from external sources. ${ }^{18}$

The invitation cover letter described the study's purpose, emphasized anonymity through aggregate-only reporting, and stated that voluntary consent was designated by responding to the survey link. The instructions reminded respondents that there were no correct or preferred opinions and that the results would be used by AASPT and ABPTRFE leadership to develop initiatives and services to promote post-professional sports physical therapy education and training. After the initial email was extended, follow-up requests were sent at one and two weeks. The survey was closed when the final invitation did not generate more than 
a $10 \%$ response increase. Before dissemination, the survey was reviewed and determined to meet exempt criteria by the Institutional Review Board at UT Southwestern Medical Center in Dallas, TX. All responses were anonymous.

\section{TOOL DEVELOPMENT}

The survey tool was initially developed by an AASPT member with 40 years of academic, residency, and sports specialty clinical experience. Previous studies with similar objectives were also used as a reference to inform survey organization and format. 1,9,14,15,19 The initial survey draft was piloted with four AASPT members familiar with postprofessional education. Their critique regarding the survey's questions, organization, and readability enhanced the face validity of the content. Based on this collective input, the survey was modified and finalized for distribution. The final data collection instrument was a 116-item questionnaire.

The general categories for data capture on the survey were divided into five sections:. For sections two through five, the survey respondent ranked each factor on an ordinal scale from 0-5 ranging from not important to extremely important.

Section 1: Twenty-five demographic questions regarding respondent's age, sex, ethnicity, marital status, geographical location, membership status, clinical experience, educational background, credentials, athletic interests, residency/fellowship involvement, and employment title and responsibilities.

Section 2: Fifteen items that solicited the applicant's motivations and faculty respondent's perception on the importance of values that motivate the pursuit of residency education and training.

Section 3: Forty-five items that solicited the applicants and faculty's opinions on the variables that influence the application and/or acceptance to a specific residency or fellowship training site.

Section 4: Eleven items regarding the importance of various methods to acquire, exchange, and disseminate specific details inherent to individual residency programs from the perspective of both applicants and providers

Section 5: Thirteen items that solicited opinions regarding factors relevant to the match and selection of a resident to a particular training program.

\section{DATA ANALYSIS}

Measures of central tendencies were derived using a spreadsheet generated from a Microsoft Excel Data Analysis, 2010 package to describe the demographic profile of the respondents. Scores on each item, regardless of section, were calculated from the sum of rating values for each question. Based on distributions of the sum, Mann-Whitney Rank Sum tests from an on-line program at www.vassarstats.net were used to detect differences between the perspectives of applicants and faculty with a significance level of $p<0.05$ being considered significant. ${ }^{20}$ Ordinal rankings of importance were created based on the median percentiles for both groups for each category of assessment. Factors characterized as "not important" were items that ranked in the bot- tom $20 \%$ percentile, mildly important in the $20-39 \%$ percentile, moderately important in the $40-59 \%$ percentile, very important in the $60-79 \%$ perecentile, and extremely important in the top $20 \%$ percentile.

\section{RESULTS}

The 156 invitations resulted in 157 responses to the survey. Fifty-seven residents or residency applicants and 50 residency/fellowship program faculty members completed the survey for a $69 \%$ known response. Fifty additional surveys were received in which the respondent indicated they had not been involved in a residency or fellowship training program as an applicant or faculty member. These responses were not used for the statistical analysis. $71 \%$ of all the respondents were male with a mean age of $33.1 \pm 9.4$. All were AASPT members and represented $88 \%$ of the states with accredited residency programs. As anticipated there was a significant difference between faculty and applicants in regards to age ( $40.0 \pm 9.6$ vs. $26.9 \pm 2.1 ; p<0.0001)$, experience ( $7.7 \pm 9.4$ vs. $0.25 \pm 0.49$ years; $p<0.0001)$, entry-level professional degree (52\% DPT vs $100 \%$ DPT), marital status (80\% vs. 26\% married), and athletic training licensure (34\% vs. $14 \%$ Athletic Trainer, Certified [ATC]). There was no difference between groups in regards to sex (74\% vs 68\% male; $p=0.62$ ), AASPT membership status (both 100\%), race/ethnicity (both $94 \%$ white of those reporting), personal competitive athletic background (both 100\%), Certified Strength and Conditioning Specialist credential (CSCS) (44\% vs 40\%) or perception in ideal clinical productivity $(30.2+12.7$ vs. $29.4+11.5$ daily units charged; $p=0.65$ ). (Table 1 )

Table 2 details the importance of the factors that may motivate the pursuit of residency education and training. Of the 15 factors surveyed, eight were rated as significantly more important to the resident than to the program faculty $(p \leqslant 0.03)$. This included seven of the 10 most important factors. Motivations that were rated as extremely important by both parties included the acquisition of clinical skills, knowledge, and critical thinking under the guidance of an accomplished mentor while fulfilling a personal passion and desire to practice in the sports physical therapy field.

Table 3 details the applicant's and faculty's opinions on what variables influence the application and/or acceptance to a specific residency or fellowship training site. Of the 45 variables surveyed there were four rated as significantly more important to the resident/fellow applicant than to the program faculty and four additional variables that were significantly more important to the program faculty than resident/fellow applicants. The only variable rated as extremely important was the potential for future job opportunities by the resident (4.32 vs $3.81 ; p<0.003$ ).

Multiple other factors were rated as very important in influencing an applicant to apply to or accept an offer from a particular program with a premium on the overall perception from the interview experience in regards to the faculty's qualifications, stability, and mentoring abilities. Additionally, the clinic infrastructure, learning opportunities, and ability to work in specific sports were highly valued. The variables rated more important to the resident than the program faculty were the future job opportunities, a preference for an academic environment with teaching oppor- 
Table 1. Demographic characteristics of survey respondents

\begin{tabular}{|c|c|c|}
\hline Demographic Characteristic & Residents/Applicants ( $n=57)$ & Directors/Faculty $(\mathrm{n}=50)$ \\
\hline Age (mean \pm SD) (range) & $26.9 \pm 2.1(24-33)$ & $40.0 \pm 9.6(29-72)$ \\
\hline Sex & 39 male; 18 female & 37 male; 13 female \\
\hline APTA member (\% yes) & $57 / 57(100 \%)$ & $50 / 50(100 \%)$ \\
\hline AASPT member (\% yes) & $57 / 57(100 \%)$ & $50 / 50(100 \%)$ \\
\hline Race & $\begin{array}{l}50 \text { White (not of Hispanic origin) } \\
4 \text { (Asian or Pacific Islander) } \\
2 \text { Hispanic/Latino } \\
1 \text { Other }\end{array}$ & $\begin{array}{l}47 \text { White (not of Hispanic origin } \\
2 \text { Hispanic/Latino } \\
1 \text { Asian or Pacific Islander }\end{array}$ \\
\hline Marital Status & $\begin{array}{l}42 \text { single }(74 \%) \\
15 \text { married }(26 \%)\end{array}$ & $\begin{array}{l}40 \text { married }(80 \%) \\
7 \text { single }(14 \%) \\
1 \text { divorced }(2 \%) \\
1 \text { widowed }(2 \%) \\
1 \text { prefer not to answer }(2 \%)\end{array}$ \\
\hline Physical Therapy School Location & 23 unique states & 21 unique states \\
\hline Experience (yrs) (mean + SD) (range) & $0.25 \pm 0.49(0-2)$ & $7.7 \pm 9.4$ \\
\hline Entry Level Degree & 57 DPT (100\%) & $\begin{array}{l}26 \text { DPT }(52 \%) \\
17 \text { Masters (34\%) } \\
6 \text { Baccalaureate } 12 \%) \\
1 \text { Certificate }(2 \%)\end{array}$ \\
\hline Highest Degree & $57 / 57$ no further degrees ( $100 \%)$ & $\begin{array}{l}42 \text { no further degrees (84\%) } \\
9 \text { tDPT (18\%) } \\
6 \text { Post-Doctoral (12\%) }\end{array}$ \\
\hline $\begin{array}{l}\text { Personal Competitive Athletic } \\
\text { Background }\end{array}$ & $57 / 57(100 \%)$ & $50 / 50(100 \%)$ \\
\hline $\begin{array}{l}\text { Youth Athlete } \\
\text { High School Athlete } \\
\text { Collegiate Athlete } \\
\text { Professional Athlete }\end{array}$ & $\begin{array}{l}43 / 57(75 \%) \\
34 / 57(60 \%) \\
31 / 57(54 \%) \\
2 / 57(4 \%)\end{array}$ & $\begin{array}{l}38 / 50(76 \%) \\
28 / 50(56 \%) \\
26 / 50(54 \%) \\
1 / 50(2 \%)\end{array}$ \\
\hline $\begin{array}{l}\text { Possess Additional Relevant } \\
\text { Certifications }\end{array}$ & $27 / 57(47 \%)$ & $45 / 50(90 \%)$ \\
\hline$\%$ of each & $\begin{array}{l}\text { 8/57 (14\%) ATC } \\
23 / 57(40 \%) \text { CSCS } \\
\text { 2/57 (3\%) OCS } \\
\text { 0/57 (0\%) FAAOMPT }\end{array}$ & $\begin{array}{l}17 / 50(34 \%) \text { ATC } \\
22 / 50(44 \%) \text { CSCS } \\
16 / 50(32 \%) \text { OCS } \\
3 / 50(6 \%) \text { FAAOMPT }\end{array}$ \\
\hline Job Title & $\begin{array}{l}\text { 36/57 (63\%) Resident/Fellow } \\
\text { 12/57 (21\%) Staff Clinician } \\
\text { 5/57 (9\%) Student Physical Therapist } \\
\text { 4/57 (7\%) Other }\end{array}$ & $\begin{array}{l}\text { 17/50 (34\%) Supervisor/Director } \\
\text { 16/50 (32\%) Staff Clinician } \\
\text { 13/50 (26\%) Faculty } \\
\text { 4/50 (8\%) Administrator/Manager/ } \\
\text { Owner }\end{array}$ \\
\hline $\begin{array}{l}\text { Number of Residency Applications } \\
\text { Submitted }\end{array}$ & $4.6+2.3$ & Not applicable \\
\hline
\end{tabular}

APTA - American Physical Therapy Association

AASPT - American Academy of Sports Physical Therapy

DPT - Doctor of Physical Therapy

tDPT - Transitional Doctor of Physical Therapy

ATC - Athletic Trainer, Certified

CSCS - Certified Strength and Conditioning Specialist

OCS - Orthopedic Clinical Specialist

FAAOMPT - Fellow of the American Academy of Orthopedic Manual Physical Therapists

tunities, and the potential for supplemental learning opportunities beyond didactic accreditation requirements $(p \leqslant$ 0.02). Conversely, there were four variables rated as significantly more important by faculty than resident/fellow applicant respondents. Two such factors that were rated as very important included the regional and/or national reputation of the program $(p=0.001)$ as well as the program's historical passing rate on the sport's specialty $(p=0.02)$ examination. Faculty also overestimated the importance of the geographical location of the residency as well as the needs, desires, and preferences of their spouse or significant other $(p \leqslant 0.01)$. Although only rated as somewhat important, faculty also assigned more importance to post-interview follow-up or contact by the program $(p=0.001)$. 
Table 2. Values that motivate the pursuit of residency education and training

\begin{tabular}{|c|c|c|c|c|c|}
\hline Parameter & Group & $\begin{array}{l}\text { Categorical } \\
\text { Ranking } \\
\text { Importance } \\
\text { Range }\end{array}$ & $\begin{array}{l}\text { Median } \\
\text { Category }\end{array}$ & Mean & $p$-value \\
\hline \multirow{2}{*}{$\begin{array}{l}\text { Gain knowledge, skills, and expertise in } \\
\text { the practice of sports physical therapy }\end{array}$} & Residents & $\mathrm{VI}-\mathrm{El}$ & El & $4.83 \pm 0.38$ & \multirow{2}{*}{0.31} \\
\hline & Faculty & SI - EI & El & $4.79 \pm 0.46$ & \\
\hline \multirow{2}{*}{$\begin{array}{l}\text { Enhance clinical reasoning and critical } \\
\text { thinking skills }\end{array}$} & Residents & VI - EI & El & $4.81 \pm 0.40$ & \multirow{2}{*}{$0.02^{*}$} \\
\hline & Faculty & $\mathrm{SI}-\mathrm{EI}$ & EI & $4.58 \pm 0.58$ & \\
\hline \multirow{2}{*}{$\begin{array}{l}\text { Fulfill passion and desire to practice } \\
\text { sports physical therapy }\end{array}$} & Residents & $\mathrm{VI}-\mathrm{EI}$ & $\mathrm{El}$ & $4.77 \pm 0.42$ & \multirow{2}{*}{0.32} \\
\hline & Faculty & $\mathrm{SI}-\mathrm{EI}$ & $\mathrm{El}$ & $4.66 \pm 0.52$ & \\
\hline \multirow{2}{*}{$\begin{array}{l}\text { Enhance the ability to examine, diagnose, } \\
\text { prognose, and improve patient outcomes }\end{array}$} & Residents & SI-EI & EI & $4.60 \pm 0.62$ & \multirow{2}{*}{$0.02^{*}$} \\
\hline & Faculty & $\mathrm{SI}-\mathrm{EI}$ & VI & $4.33 \pm 0.69$ & \\
\hline \multirow{2}{*}{$\begin{array}{l}\text { Enhance career advancement and future } \\
\text { job opportunities }\end{array}$} & Residents & $\mathrm{MI}-\mathrm{El}$ & $\mathrm{EI}$ & $4.56 \pm 0.71$ & \multirow{2}{*}{$0.02^{*}$} \\
\hline & Faculty & $\mathrm{SI}-\mathrm{EI}$ & VI & $4.31 \pm 0.68$ & \\
\hline \multirow{2}{*}{$\begin{array}{l}\text { Access to an accomplished mentor to } \\
\text { provide feedback and boost confidence }\end{array}$} & Residents & MI-EI & EI & $4.52 \pm 0.73$ & \multirow{2}{*}{0.33} \\
\hline & Faculty & $\mathrm{MI}-\mathrm{EI}$ & El & $4.54 \pm 0.62$ & \\
\hline \multirow{2}{*}{$\begin{array}{l}\text { Enhance the ability to use current best } \\
\text { evidence patient management strategies }\end{array}$} & Residents & $\mathrm{SI}-\mathrm{EI}$ & EI & $4.51 \pm 0.66$ & \multirow{2}{*}{$0.03^{*}$} \\
\hline & Faculty & SI-EI & VI & $4.29 \pm 0.69$ & \\
\hline \multirow{2}{*}{$\begin{array}{l}\text { Enhance future leadership opportunities } \\
\text { within the field of sports physical therapy }\end{array}$} & Residents & $\mathrm{MI}-\mathrm{El}$ & VI & $4.12 \pm 0.81$ & \multirow{2}{*}{$0.001^{*}$} \\
\hline & Faculty & $\mathrm{MI}-\mathrm{El}$ & VI & $3.76 \pm 0.72$ & \\
\hline \multirow{2}{*}{$\begin{array}{l}\text { Validate a commitment to lifelong } \\
\text { learning }\end{array}$} & Residents & $\mathrm{NI}-\mathrm{El}$ & VI & $4.09 \pm 0.1 .08$ & \multirow{2}{*}{$0.01^{*}$} \\
\hline & Faculty & $\mathrm{MI}-\mathrm{EI}$ & VI & $3.73 \pm 0.96$ & \\
\hline \multirow{2}{*}{$\begin{array}{l}\text { Contribute to the evolution of the } \\
\text { physical therapy profession }\end{array}$} & Residents & $\mathrm{NI}-\mathrm{EI}$ & VI & $4.04 \pm 0.93$ & \multirow{2}{*}{$0.02^{*}$} \\
\hline & Faculty & $\mathrm{NI}-\mathrm{EI}$ & VI & $3.54 \pm 0.90$ & \\
\hline \multirow{2}{*}{$\begin{array}{l}\text { Fast track to sports specialization } \\
\text { credential }\end{array}$} & Residents & $\mathrm{NI}-\mathrm{El}$ & $\mathrm{VI}$ & $3.60 \pm 0.1 .35$ & \multirow{2}{*}{0.18} \\
\hline & Faculty & MI-EI & VI & $4.09 \pm 0.86$ & \\
\hline \multirow{2}{*}{$\begin{array}{l}\text { Enhance the ability to conduct and } \\
\text { interpret research }\end{array}$} & Residents & $\mathrm{NI}-\mathrm{El}$ & SI & $3.47 \pm 0.97$ & \multirow{2}{*}{$0.05^{*}$} \\
\hline & Faculty & MI-EI & SI & $3.20 \pm 0.71$ & \\
\hline \multirow{2}{*}{ Enhance the potential for future income } & Residents & $\mathrm{NI}-\mathrm{EI}$ & $\mathrm{SI}$ & $3.40 \pm 0.1 .10$ & \multirow{2}{*}{0.32} \\
\hline & Faculty & $\mathrm{MI}-\mathrm{EI}$ & $\mathrm{SI}$ & $3.41 \pm 0.89$ & \\
\hline \multirow{2}{*}{$\begin{array}{l}\text { Gain recognition from physicians or other } \\
\text { types of sports healthcare providers }\end{array}$} & Residents & $\mathrm{NI}-\mathrm{EI}$ & SI & $3.23 \pm 1.12$ & \multirow{2}{*}{0.35} \\
\hline & Faculty & $\mathrm{MI}-\mathrm{El}$ & $\mathrm{SI}$ & $3.29 \pm 0.89$ & \\
\hline \multirow{2}{*}{$\begin{array}{l}\text { Gain recognition from other physical } \\
\text { therapists }\end{array}$} & Residents & $\mathrm{NI}-\mathrm{EI}$ & $\mathrm{SI}$ & $2.93 \pm 1.09$ & \multirow{2}{*}{0.31} \\
\hline & Faculty & $\mathrm{MI}-\mathrm{EI}$ & SI & $3.20 \pm 0.76$ & \\
\hline
\end{tabular}

EI: Extremely important VI: Very Important SI: Somewhat Important MI: Mildly Important NI: Not Important $* p \leqslant 0.05$

Table 4 details the perceived value of 11 methods to acquire exchange and disseminate information about residency and fellowship programs. Both cohorts found the interview day to be the most important mechanism to reveal specific details inherent to individual residency programs. Other areas rated as very important included access to the program director's phone number and email contact, web site content, RF-PTCAS synopsis and links, and potential contact with previous or current residents or fellows. The opportunity for applicants to contact previous or current residents was rated significantly higher by faculty than applicants $(p=0.04)$. Providing printed materials with resi- dency program information was rated significantly lower by the applicant than the faculty $(p=0.04)$.

The most consistent area of agreement between faculty and applicant respondents was in the area of factors that are important to the match and selection of a resident to a particular training program. Two areas that were rated as extremely important by both groups were the interview performance and letters of recommendation. There was also concurrence at the other end of the importance spectrum with the past and future geographical location preferences rated as minimally important. The one area in which there was a difference in opinion was the perception of the like- 
Table 3. Variables that influence the application and/or acceptance to a specific residency or fellowship training site

\begin{tabular}{|c|c|c|c|c|c|}
\hline Parameter & Group & $\begin{array}{l}\text { Categorical } \\
\text { Ranking } \\
\text { Importance } \\
\text { Range }\end{array}$ & $\begin{array}{l}\text { Median } \\
\text { Category }\end{array}$ & Mean & $p$-value \\
\hline \multirow{2}{*}{ Future job opportunities } & Residents & $\mathrm{MI}-\mathrm{EI}$ & $\mathrm{El}$ & $4.32 \pm 1.04$ & \multirow{2}{*}{$0.003^{*}$} \\
\hline & Faculty & $\mathrm{MI}-\mathrm{EI}$ & VI & $3.81 \pm 0.94$ & \\
\hline \multirow{2}{*}{$\begin{array}{l}\text { Perceived relationship between resident(s) and } \\
\text { program director/faculty }\end{array}$} & Residents & $\mathrm{MI}-\mathrm{El}$ & VI & $4.30 \pm 0.85$ & \multirow{2}{*}{0.2} \\
\hline & Faculty & $\mathrm{MI}-\mathrm{EI}$ & VI & $4.19 \pm 0.70$ & \\
\hline \multirow{2}{*}{$\begin{array}{l}\text { Impression and qualifications of residency } \\
\text { program faculty and clinical personnel }\end{array}$} & Residents & $\mathrm{MI}-\mathrm{El}$ & VI & $4.05+0.72$ & \multirow{2}{*}{0.27} \\
\hline & Faculty & $\mathrm{MI}-\mathrm{EI}$ & VI & $4.21 \pm 071$ & \\
\hline \multirow{2}{*}{ Advice from trusted mentor or colleague } & Residents & $\mathrm{MI}-\mathrm{EI}$ & VI & $4.04 \pm 0.80$ & \multirow{2}{*}{0.18} \\
\hline & Faculty & $\mathrm{MI}-\mathrm{EI}$ & $\mathrm{VI}$ & $4.27 \pm 0.71$ & \\
\hline \multirow{2}{*}{ Overall interview experience } & Residents & $\mathrm{MI}-\mathrm{El}$ & VI & $4.00 \pm 0.87$ & \multirow{2}{*}{0.32} \\
\hline & Faculty & $\mathrm{MI}-\mathrm{EI}$ & VI & $4.19 \pm 0.64$ & \\
\hline \multirow{2}{*}{$\begin{array}{l}\text { Perceived stability of department or clinic that is } \\
\text { sponsoring residency program }\end{array}$} & Residents & $\mathrm{NI}-\mathrm{El}$ & VI & $3.96 \pm 1.03$ & \multirow{2}{*}{0.06} \\
\hline & Faculty & $\mathrm{NI}-\mathrm{EI}$ & VI & $3.79 \pm 0.98$ & \\
\hline \multirow{2}{*}{$\begin{array}{l}\text { Additional or supplemental learning opportunities } \\
\text { made available and/or required (continuing ed } \\
\text { classes, grand rounds, journal clubs, etc) }\end{array}$} & Residents & $\mathrm{MI}-\mathrm{EI}$ & VI & $3.93 \pm 1.05$ & \multirow{2}{*}{$0.02^{*}$} \\
\hline & Faculty & $\mathrm{NI}-\mathrm{El}$ & VI & $3.66 \pm 0.89$ & \\
\hline \multirow{2}{*}{$\begin{array}{l}\text { Impression and qualifications of residency } \\
\text { program director }\end{array}$} & Residents & $\mathrm{NI}-\mathrm{El}$ & VI & $3.86 \pm 0.83$ & \multirow{2}{*}{0.49} \\
\hline & Faculty & $\mathrm{MI}-\mathrm{El}$ & VI & $3.94 \pm 0.86$ & \\
\hline \multirow{2}{*}{$\begin{array}{l}\text { Personal interactions (in person or electronic) } \\
\text { with previous and/or current residents }\end{array}$} & Residents & $\mathrm{NI}-\mathrm{EI}$ & VI & $3.84 \pm 1.08$ & \multirow{2}{*}{0.06} \\
\hline & Faculty & MI-EI & VI & $3.73 \pm 0.68$ & \\
\hline \multirow{2}{*}{$\begin{array}{l}\text { Clinic infrastructure, organization, space, and } \\
\text { equipment }\end{array}$} & Residents & $\mathrm{Ml}-\mathrm{EI}$ & VI & $3.82 \pm 0.95$ & \multirow{2}{*}{0.40} \\
\hline & Faculty & MI-El & VI & $3.98 \pm 0.73$ & \\
\hline \multirow{2}{*}{$\begin{array}{l}\text { Access to and/or interaction with physicians } \\
\text { (clinics, rounds, surgery observation, etc) }\end{array}$} & Residents & $\mathrm{NI}-\mathrm{EI}$ & VI & $3.82 \pm 0.91$ & \multirow{2}{*}{0.26} \\
\hline & Faculty & $\mathrm{MI}-\mathrm{El}$ & VI & $3.77 \pm 0.90$ & \\
\hline \multirow{2}{*}{$\begin{array}{l}\text { Opportunity to work with a specific type of sport } \\
\text { or activity }\end{array}$} & Residents & $\mathrm{NI}-\mathrm{EI}$ & VI & $3.79 \pm 1.25$ & \multirow{2}{*}{0.29} \\
\hline & Faculty & $\mathrm{Ml}-\mathrm{El}$ & VI & $4.06 \pm 0.92$ & \\
\hline \multirow{2}{*}{$\begin{array}{l}\text { Access to and/or interaction with other health } \\
\text { care professionals }\end{array}$} & Residents & $\mathrm{NI}-\mathrm{EI}$ & $\mathrm{VI}$ & $3.79 \pm 0.98$ & \multirow{2}{*}{0.18} \\
\hline & Faculty & MI-EI & $\mathrm{VI}$ & $3.71 \pm 0.90$ & \\
\hline Residency is designed or embedded within an & Residents & $\mathrm{NI}-\mathrm{EI}$ & VI & $3.79 \pm 1.26$ & $0001 *$ \\
\hline academic environment or university setting & Faculty & $\mathrm{NI}-\mathrm{El}$ & $\mathrm{SI}$ & $3.17 \pm 1.12$ & 0.001 \\
\hline The format, methods, and content of the didactic & Residents & MI - El & VI & $3.67 \pm 0.87$ & 027 \\
\hline curriculum that accompanies the residency & Faculty & $\mathrm{NI}-\mathrm{EI}$ & VI & $3.65 \pm 0.96$ & ונד \\
\hline Opportunities for teaching physical therapy & Residents & $\mathrm{NI}-\mathrm{El}$ & VI & $3.65 \pm 1.33$ & \\
\hline $\begin{array}{l}\text { students or other health care protessionals durıng } \\
\text { the residency program }\end{array}$ & Faculty & $\mathrm{NI}-\mathrm{El}$ & SI & $3.34 \pm 0.98$ & 0.02 \\
\hline 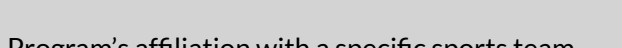 & Residents & $\mathrm{NI}-\mathrm{EI}$ & VI & $3.58 \pm 1.13$ & 021 \\
\hline Program s armilation witn a specinc sports team & Faculty & $\mathrm{NI}-\mathrm{El}$ & VI & $3.96 \pm 0.81$ & 0.24 \\
\hline Reginnal and/or national renutation of nrogram & Residents & $\mathrm{NI}-\mathrm{El}$ & $\mathrm{VI}$ & $3.56 \pm 1.05$ & $0001^{*}$ \\
\hline 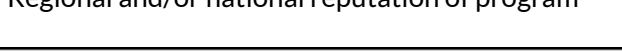 & Faculty & $\mathrm{MI}-\mathrm{EI}$ & VI & $4.17 \pm 0.83$ & 0.001 \\
\hline Patient caseload diagnostic diversity and/or & Residents & $\mathrm{NI}-\mathrm{EI}$ & VI & $3.49 \pm 1.18$ & 021 \\
\hline emphasis & Faculty & $\mathrm{NI}-\mathrm{El}$ & VI & $3.56 \pm 0.92$ & 0.34 \\
\hline Perceived camaraderie or current or past & Residents & $\mathrm{NI}-\mathrm{El}$ & $\mathrm{VI}$ & $3.38 \pm 1.09$ & 015 \\
\hline residents & Faculty & $\mathrm{NI}-\mathrm{El}$ & $\mathrm{SI}$ & $3.46 \pm 0.74$ & 0.4 \\
\hline
\end{tabular}




\begin{tabular}{|c|c|c|c|c|c|}
\hline Parameter & Group & $\begin{array}{l}\text { Categorical } \\
\text { Ranking } \\
\text { Importance } \\
\text { Range }\end{array}$ & $\begin{array}{l}\text { Median } \\
\text { Category }\end{array}$ & Mean & $p$-value \\
\hline \multirow{2}{*}{$\begin{array}{l}\text { Program's historical passing rate for the sports } \\
\text { specialty exam }\end{array}$} & Residents & $\mathrm{NI}-\mathrm{El}$ & VI & $3.35 \pm 1.32$ & \multirow{2}{*}{$0.02^{*}$} \\
\hline & Faculty & $\mathrm{NI}-\mathrm{EI}$ & VI & $4.00 \pm 1.08$ & \\
\hline \multirow{2}{*}{$\begin{array}{l}\text { Opportunities for participating in research } \\
\text { activities during the residency program }\end{array}$} & Residents & $\mathrm{NI}-\mathrm{EI}$ & $\mathrm{SI}$ & $3.19 \pm 1.23$ & \multirow{2}{*}{0.34} \\
\hline & Faculty & $\mathrm{NI}-\mathrm{El}$ & $\mathrm{SI}$ & $3.17 \pm 0.88$ & \\
\hline \multirow{2}{*}{$\begin{array}{l}\text { Projected daily/weekly caseload (productivity } \\
\text { expectation }\end{array}$} & Residents & $\mathrm{NI}-\mathrm{El}$ & $\mathrm{SI}$ & $3.09 \pm 1.24$ & \multirow{2}{*}{0.17} \\
\hline & Faculty & $\mathrm{NI}-\mathrm{EI}$ & SI & $3.35 \pm 0.84$ & \\
\hline \multirow{2}{*}{$\begin{array}{l}\text { Clinic hours (daily schedule, hours of operation, } \\
\text { hours/week of work, etc) }\end{array}$} & Residents & $\mathrm{NI}-\mathrm{El}$ & $\mathrm{SI}$ & $3.05 \pm 1.26$ & \multirow{2}{*}{0.49} \\
\hline & Faculty & $\mathrm{NI}-\mathrm{El}$ & $\mathrm{SI}$ & $3.19 \pm 0.89$ & \\
\hline \multirow{2}{*}{ Salary offered by residency program } & Residents & $\mathrm{NI}-\mathrm{El}$ & $\mathrm{SI}$ & $3.05 \pm 1.06$ & \multirow{2}{*}{0.12} \\
\hline & Faculty & $\mathrm{MI}-\mathrm{El}$ & $\mathrm{SI}$ & $3.35 \pm 0.81$ & \\
\hline \multirow{2}{*}{$\begin{array}{l}\text { Benefits package available to residents (insurance, } \\
\text { retirement plan, continuing education, vacation, } \\
\text { sick leave, etc) }\end{array}$} & Residents & $\mathrm{NI}-\mathrm{EI}$ & $\mathrm{SI}$ & $3.04 \pm 1.21$ & \multirow{2}{*}{0.12} \\
\hline & Faculty & $\mathrm{MI}-\mathrm{EI}$ & $\mathrm{SI}$ & $3.38 \pm 0.82$ & \\
\hline \multirow{2}{*}{ Geographic location of the residency } & Residents & $\mathrm{NI}-\mathrm{EI}$ & $\mathrm{SI}$ & $3.04 \pm 1.40$ & \multirow{2}{*}{$0.01^{*}$} \\
\hline & Faculty & $\mathrm{MI}-\mathrm{El}$ & $\mathrm{SI}$ & $3.53 \pm 0.86$ & \\
\hline \multirow{2}{*}{$\begin{array}{l}\text { Extent and availability of library and professional } \\
\text { journal resources }\end{array}$} & Residents & $\mathrm{NI}-\mathrm{EI}$ & $\mathrm{SI}$ & $2.98 \pm 1.27$ & \multirow{2}{*}{0.25} \\
\hline & Faculty & $\mathrm{NI}-\mathrm{El}$ & $\mathrm{SI}$ & $2.88 \pm 1.02$ & \\
\hline \multirow{2}{*}{$\begin{array}{l}\text { Residency is designed or embedded within a } \\
\text { clinical environment in the community }\end{array}$} & Residents & $\mathrm{NI}-\mathrm{EI}$ & SI & $2.96 \pm 1.30$ & \multirow{2}{*}{0.26} \\
\hline & Faculty & $\mathrm{NI}-\mathrm{El}$ & $\mathrm{SI}$ & $2.96 \pm 0.97$ & \\
\hline \multirow{2}{*}{$\begin{array}{l}\text { Placement in subsequent fellowship or advanced } \\
\text { training programs }\end{array}$} & Residents & $\mathrm{NI}-\mathrm{EI}$ & $\mathrm{SI}$ & $2.96 \pm 1.46$ & \multirow{2}{*}{0.37} \\
\hline & Faculty & $\mathrm{NI}-\mathrm{El}$ & $\mathrm{SI}$ & $2.93 \pm 1.35$ & \\
\hline \multirow{2}{*}{$\begin{array}{l}\text { Needs, desires, or preferences of spouse or } \\
\text { significant other }\end{array}$} & Residents & $\mathrm{NI}-\mathrm{El}$ & $\mathrm{SI}$ & $2.95 \pm 1.29$ & \multirow{2}{*}{$0.001^{*}$} \\
\hline & Faculty & $\mathrm{NI}-\mathrm{EI}$ & $\mathrm{SI}$ & $3.30 \pm 1.12$ & \\
\hline \multirow{2}{*}{$\begin{array}{l}\text { Perceived favorable training environment for } \\
\text { women }\end{array}$} & Residents & $\mathrm{NI}-\mathrm{El}$ & SI & $2.93 \pm 1.77$ & \multirow{2}{*}{0.14} \\
\hline & Faculty & $\mathrm{NI}-\mathrm{El}$ & $\mathrm{SI}$ & $3.23 \pm 1.48$ & \\
\hline \multirow{2}{*}{$\begin{array}{l}\text { Perceived favorable training environment for } \\
\text { minorities }\end{array}$} & Residents & $\mathrm{NI}-\mathrm{EI}$ & SI & $2.93 \pm 1.77$ & 11 \\
\hline & Faculty & $\mathrm{NI}-\mathrm{EI}$ & $\mathrm{SI}$ & $3.21 \pm 1.48$ & 0.11 \\
\hline Residency is designed in a collaborative model & Residents & $\mathrm{NI}-\mathrm{El}$ & $\mathrm{SI}$ & $2.92 \pm 1.60$ & \\
\hline $\begin{array}{l}\text { clinic partner(s). } \\
\text { colition }\end{array}$ & Faculty & $\mathrm{NI}-\mathrm{El}$ & MI & $2.61 \pm 1.27$ & 0.22 \\
\hline I noth of recidoncy trainino nrorrom & Residents & $\mathrm{NI}-\mathrm{El}$ & $\mathrm{SI}$ & $2.91 \pm 1.25$ & 021 \\
\hline Lengtn or resiaency training program & Faculty & $\mathrm{NI}-\mathrm{El}$ & $\mathrm{SI}$ & $3.28 \pm 0.80$ & 0.24 \\
\hline Characteristics of the area in which the residency & Residents & $\mathrm{NI}-\mathrm{El}$ & $\mathrm{SI}$ & $2.84 \pm 1.21$ & \\
\hline $\begin{array}{l}\text { Is located (urban vs suburban vs rural, social } \\
\text { atmosphere, recreational opportunities, etc) }\end{array}$ & Faculty & $\mathrm{NI}-\mathrm{El}$ & $\mathrm{SI}$ & $3.00 \pm 0.92$ & 0.43 \\
\hline Post-interview follow-up or contact by the & Residents & $\mathrm{NI}-\mathrm{EI}$ & MI & $2.49 \pm 1.29$ & $0 \cap \Omega^{*}$ \\
\hline program (perceived likelihood of acceptance) & Faculty & $\mathrm{NI}-\mathrm{EI}$ & $\mathrm{SI}$ & $3.04 \pm 1.09$ & $0.04^{*}$ \\
\hline Cost of living in the city where the residency & Residents & $\mathrm{NI}-\mathrm{El}$ & $\mathrm{SI}$ & $2.40 \pm 1.13$ & $0001 *$ \\
\hline resides & Faculty & $\mathrm{NI}-\mathrm{El}$ & $\mathrm{SI}$ & $2.96 \pm 0.74$ & 0.001 \\
\hline Emphasis on local, state, and/or national APTA & Residents & $\mathrm{NI}-\mathrm{El}$ & MI & $2.39 \pm 1.05$ & 041 \\
\hline membership and involvement & Faculty & $\mathrm{NI}-\mathrm{EI}$ & MI & $2.46 \pm 0.94$ & 0.41 \\
\hline Residency accepts at least two residents in each & Residents & $\mathrm{NI}-\mathrm{El}$ & MI & $2.35 \pm 1.52$ & 046 \\
\hline cohort & Faculty & $\mathrm{NI}-\mathrm{El}$ & MI & $2.55 \pm 1.39$ & 0.40 \\
\hline Opportunity to pursue additional degrees or & Residents & $\mathrm{NI}-\mathrm{EI}$ & MI & $2.13 \pm 1.26$ & 045 \\
\hline certifications at the institution & Faculty & $\mathrm{NI}-\mathrm{El}$ & MI & $2.33 \pm 1.16$ & 0.45 \\
\hline
\end{tabular}




\begin{tabular}{|c|c|c|c|c|c|}
\hline Parameter & Group & $\begin{array}{l}\text { Categorical } \\
\text { Ranking } \\
\text { Importance } \\
\text { Range }\end{array}$ & $\begin{array}{l}\text { Median } \\
\text { Category }\end{array}$ & Mean & $p$-value \\
\hline \multirow{2}{*}{$\begin{array}{l}\text { Tolerance and allowances regarding remediation } \\
\text { policies }\end{array}$} & Residents & $\mathrm{NI}-\mathrm{EI}$ & $\mathrm{NI}$ & $2.12 \pm 1.39$ & \multirow{2}{*}{0.17} \\
\hline & Faculty & $\mathrm{NI}-\mathrm{EI}$ & MI & $2.15 \pm 0.99$ & \\
\hline \multirow{2}{*}{$\begin{array}{l}\text { Opportunity, availability, and/or allowance for } \\
\text { supplemental moonlighting work }\end{array}$} & Residents & $\mathrm{NI}-\mathrm{EI}$ & $\mathrm{NI}$ & $1.77 \pm 1.18$ & \multirow{2}{*}{0.25} \\
\hline & Faculty & $\mathrm{NI}-\mathrm{EI}$ & MI & $1.95 \pm 1.10$ & \\
\hline \multirow{2}{*}{$\begin{array}{l}\text { Residency accepts only one resident for each } \\
\text { cohort }\end{array}$} & Residents & $\mathrm{NI}-\mathrm{El}$ & $\mathrm{NI}$ & $1.47 \pm 0.98$ & \multirow{2}{*}{0.12} \\
\hline & Faculty & $\mathrm{NI}-\mathrm{El}$ & MI & $1.95 \pm 1.14$ & \\
\hline \multirow{2}{*}{$\begin{array}{l}\text { Opportunity for part-time and/or reduced-pace } \\
\text { program }\end{array}$} & Residents & $\mathrm{NI}-\mathrm{EI}$ & $\mathrm{NI}$ & $1.38 \pm 0.95$ & \multirow[b]{2}{*}{0.18} \\
\hline & Faculty & $\mathrm{NI}-\mathrm{EI}$ & $\mathrm{MI}$ & $1.74 \pm 1.13$ & \\
\hline
\end{tabular}

EI: Extremely important VI: Very Important SI: Somewhat Important MI: Mildly Important NI: Not Important * $p \leqslant 0.05$

Table 4. Importance of various methods to acquire, exchange and disseminate residency program information

\begin{tabular}{|c|c|c|c|c|c|}
\hline Parameter & Group & $\begin{array}{l}\text { Categorical Ranking } \\
\text { Importance Range }\end{array}$ & $\begin{array}{l}\text { Median } \\
\text { Category }\end{array}$ & Mean + SD & $p$-value \\
\hline \multirow{2}{*}{ Interview Day } & Residents & MI-EI & $\mathrm{EI}$ & $4.45 \pm 0.74$ & \multirow{2}{*}{0.41} \\
\hline & Faculty & $\mathrm{MI}-\mathrm{EI}$ & El & $4.40 \pm 0.84$ & \\
\hline \multirow{2}{*}{$\begin{array}{l}\text { Email contact with program } \\
\text { director and/or faculty }\end{array}$} & Residents & $\mathrm{NI}-\mathrm{EI}$ & VI & $4.11 \pm 0.99$ & \multirow{2}{*}{0.16} \\
\hline & Faculty & $\mathrm{MI}-\mathrm{El}$ & VI & $4.30 \pm 0.72$ & \\
\hline \multirow{2}{*}{$\begin{array}{l}\text { Phone contact with program } \\
\text { director and/or faculty }\end{array}$} & Residents & $\mathrm{MI}-\mathrm{El}$ & VI & $4.06 \pm 1.13$ & \multirow{2}{*}{0.18} \\
\hline & Faculty & $\mathrm{MI}-\mathrm{EI}$ & VI & $4.27 \pm 0.79$ & \\
\hline \multirow{2}{*}{ Program's Website } & Residents & $\mathrm{NI}-\mathrm{El}$ & VI & $3.93 \pm 1.02$ & \multirow{2}{*}{0.27} \\
\hline & Faculty & $\mathrm{MI}-\mathrm{El}$ & VI & $3.92 \pm 0.77$ & \\
\hline \multirow{2}{*}{$\begin{array}{l}\text { Contact with previous/current } \\
\text { residents }\end{array}$} & Residents & $\mathrm{NI}-\mathrm{EI}$ & VI & $3.80 \pm 1.12$ & \multirow{2}{*}{$0.04^{*}$} \\
\hline & Faculty & $\mathrm{MI}-\mathrm{EI}$ & VI & $4.30 \pm 0.71$ & \\
\hline \multirow{2}{*}{ RFPTCAS information and links } & Residents & $\mathrm{NI}-\mathrm{EI}$ & VI & $3.61 \pm 1.12$ & \multirow{2}{*}{0.46} \\
\hline & Faculty & $\mathrm{MI}-\mathrm{El}$ & $\mathrm{VI}$ & $3.71 \pm 0.80$ & \\
\hline \multirow{2}{*}{$\begin{array}{l}\text { Participation in the match day } \\
\text { notification process }\end{array}$} & Residents & $\mathrm{NI}-\mathrm{EI}$ & $\mathrm{SI}$ & $3.24 \pm 1.58$ & \multirow{2}{*}{0.17} \\
\hline & Faculty & $\mathrm{NI}-\mathrm{EI}$ & SI & $2.69 \pm 1.40$ & \\
\hline \multirow{2}{*}{ CSM TeamMates reception } & Residents & $\mathrm{NI}-\mathrm{EI}$ & $\mathrm{SI}$ & $2.80 \pm 1.28$ & \multirow{2}{*}{0.47} \\
\hline & Faculty & $\mathrm{NI}-\mathrm{EI}$ & SI & $2.80 \pm 1.13$ & \\
\hline \multirow{2}{*}{$\begin{array}{l}\text { Social Media (Facebook, Twitter, } \\
\text { etc) }\end{array}$} & Residents & $\mathrm{NI}-\mathrm{EI}$ & SI & $2.50 \pm 1.14$ & \multirow{2}{*}{0.14} \\
\hline & Faculty & $\mathrm{NI}-\mathrm{EI}$ & SI & $2.91 \pm 1.05$ & \\
\hline \multirow{2}{*}{$\begin{array}{l}\text { Printed Materials or Brochures } \\
\text { from the program }\end{array}$} & Residents & $\mathrm{NI}-\mathrm{EI}$ & MI & $2.31 \pm 1.26$ & \multirow{2}{*}{$0.04^{*}$} \\
\hline & Faculty & $\mathrm{NI}-\mathrm{EI}$ & SI & $2.64 \pm 0.89$ & \\
\hline \multirow{2}{*}{$\begin{array}{l}\text { Online blogs, internet sites, chat } \\
\text { rooms }\end{array}$} & Residents & $\mathrm{NI}-\mathrm{EI}$ & MI & $2.18 \pm 1.21$ & \multirow{2}{*}{0.30} \\
\hline & Faculty & $\mathrm{NI}-\mathrm{EI}$ & SI & $2.43 \pm 0.89$ & \\
\hline
\end{tabular}

EI: Extremely important VI: Very Important SI: Somewhat Important MI: Mildly Important NI: Not Important * $p \leqslant 0.05$

lihood of post-residency employment retention as being an influential factor in decision-making $(p=0.01)$ (Table 5)

\section{DISCUSSION}

The results of the survey give preliminary insights into the 
Table 5. Factors and criterion that are important to the match and selection of a resident to a specific residency/ fellowship program.

\begin{tabular}{|c|c|c|c|c|c|}
\hline Parameter & Group & $\begin{array}{c}\text { Categorical } \\
\text { Ranking } \\
\text { Importance Range }\end{array}$ & $\begin{array}{l}\text { Median } \\
\text { Category }\end{array}$ & Mean & $p$-value \\
\hline \multirow{2}{*}{ Interview performance } & Residents & $\mathrm{SI}-\mathrm{EI}$ & El & $4.70 \pm 0.50$ & \multirow{2}{*}{0.32} \\
\hline & Faculty & $\mathrm{SI}-\mathrm{EI}$ & $\overline{E l}$ & $4.78 \pm 0.47$ & \\
\hline \multirow{2}{*}{ Letters of recommendation } & Residents & $\mathrm{MI}-\mathrm{El}$ & El & $4.47 \pm 0.66$ & \multirow{2}{*}{0.15} \\
\hline & Faculty & SI - EI & El & $4.33 \pm 0.75$ & \\
\hline \multirow{2}{*}{ Future career goals } & Residents & $\mathrm{MI}-\mathrm{El}$ & VI & $4.28 \pm 0.82$ & \multirow{2}{*}{0.13} \\
\hline & Faculty & $\mathrm{MI}-\mathrm{EI}$ & $\mathrm{VI}$ & $4.12 \pm 0.86$ & \\
\hline \multirow{2}{*}{ Content of application essay(s) } & Residents & $\mathrm{MI}-\mathrm{El}$ & VI & $4.21 \pm 0.75$ & \multirow{2}{*}{0.37} \\
\hline & Faculty & $\mathrm{MI}-\mathrm{EI}$ & VI & $4.18 \pm 0.78$ & \\
\hline \multirow{2}{*}{$\begin{array}{l}\text { PT school clinical rotation and/or previous } \\
\text { job performance }\end{array}$} & Residents & $\mathrm{MI}-\mathrm{EI}$ & VI & $3.84 \pm 0.77$ & \multirow{2}{*}{0.35} \\
\hline & Faculty & $\mathrm{MI}-\mathrm{EI}$ & VI & $3.80 \pm 0.89$ & \\
\hline \multirow{2}{*}{$\begin{array}{l}\text { Previous certifications, licenses, } \\
\text { credentials, or specific work experience }\end{array}$} & Residents & $\mathrm{MI}-\mathrm{El}$ & VI & $3.67 \pm 0.91$ & \multirow{2}{*}{0.43} \\
\hline & Faculty & $\mathrm{NI}-\mathrm{El}$ & VI & $3.69 \pm 0.87$ & \\
\hline \multirow{2}{*}{$\begin{array}{l}\text { Previous relationship with program and/or } \\
\text { faculty }\end{array}$} & Residents & $\mathrm{NI}-\mathrm{El}$ & $\mathrm{VI}$ & $3.54 \pm 1.10$ & \multirow{2}{*}{0.28} \\
\hline & Faculty & $\mathrm{NI}-\mathrm{El}$ & $\mathrm{VI}$ & $3.49 \pm 1.06$ & \\
\hline \multirow{2}{*}{$\begin{array}{l}\text { Past research accomplishments and/or } \\
\text { expressed interest in conducting research }\end{array}$} & Residents & $\mathrm{MI}-\mathrm{El}$ & $\mathrm{SI}$ & $3.33 \pm 0.81$ & \multirow{2}{*}{0.24} \\
\hline & Faculty & $\mathrm{NI}-\mathrm{El}$ & $\mathrm{SI}$ & $3.25 \pm 0.78$ & \\
\hline \multirow{2}{*}{$\begin{array}{l}\text { Reputation of physical therapy school } \\
\text { attended }\end{array}$} & Residents & $\mathrm{NI}-\mathrm{EI}$ & $\mathrm{SI}$ & $3.21 \pm 1.08$ & \multirow{2}{*}{0.06} \\
\hline & Faculty & $\mathrm{NI}-\mathrm{El}$ & $\mathrm{SI}$ & $2.98 \pm 0.90$ & \\
\hline \multirow{2}{*}{$\begin{array}{l}\text { Involvement in local, state, or national } \\
\text { professional organizations }\end{array}$} & Residents & $\mathrm{MI}-\mathrm{EI}$ & $\mathrm{SI}$ & $3.19 \pm 0.81$ & \multirow{2}{*}{0.45} \\
\hline & Faculty & $\mathrm{MI}-\mathrm{El}$ & $\mathrm{SI}$ & $3.30 \pm 0.75$ & \\
\hline \multirow{2}{*}{$\begin{array}{l}\text { Previous continuing education } \\
\text { experiences }\end{array}$} & Residents & $\mathrm{NI}-\mathrm{El}$ & $\mathrm{SI}$ & $3.07 \pm 0.87$ & \multirow{2}{*}{0.26} \\
\hline & Faculty & $\mathrm{NI}-\mathrm{VI}$ & $\mathrm{SI}$ & $2.96 \pm 0.79$ & \\
\hline \multirow{2}{*}{$\begin{array}{l}\text { Likelihood of employment retention post } \\
\text { residency }\end{array}$} & Residents & $\mathrm{NI}-\mathrm{EI}$ & $\mathrm{SI}$ & $2.98 \pm 1.37$ & \multirow{2}{*}{$0.01^{*}$} \\
\hline & Faculty & $\mathrm{NI}-\mathrm{El}$ & MI & $2.41 \pm 1.15$ & \\
\hline \multirow{2}{*}{$\begin{array}{l}\text { PT school class rank (GPA and/or } \\
\text { transcript findings) }\end{array}$} & Residents & $\mathrm{NI}-\mathrm{El}$ & $\mathrm{SI}$ & $2.88 \pm 1.10$ & \multirow{2}{*}{0.11} \\
\hline & Faculty & $\mathrm{NI}-\mathrm{VI}$ & $\mathrm{SI}$ & $2.67 \pm 0.94$ & \\
\hline \multirow{2}{*}{ Pro bono or community service record } & Residents & $\mathrm{NI}-\mathrm{El}$ & $\mathrm{SI}$ & $2.79 \pm 0.94$ & \multirow{2}{*}{0.49} \\
\hline & Faculty & $\mathrm{NI}-\mathrm{EI}$ & $\mathrm{SI}$ & $2.82 \pm 0.78$ & \\
\hline \multirow{2}{*}{$\begin{array}{l}\text { Geographical location preference for } \\
\text { future employment }\end{array}$} & Residents & $\mathrm{NI}-\mathrm{EI}$ & $\mathrm{SI}$ & $2.64 \pm 1.23$ & \multirow{2}{*}{0.08} \\
\hline & Faculty & $\mathrm{NI}-\mathrm{EI}$ & MI & $2.33 \pm 1.11$ & \\
\hline & Residents & $\mathrm{NI}-\mathrm{El}$ & MI & $2.04 \pm 1.21$ & \\
\hline Geographical background/heritage & Faculty & $\mathrm{MI}-\mathrm{VI}$ & $\mathrm{MI}$ & $1.73 \pm 0.82$ & 0.12 \\
\hline
\end{tabular}

EI: Extremely important VI: Very Important SI: Somewhat Important MI: Mildly Important NI: Not Important $* p \leqslant 0.05$

attributes, attitudes, motivations, and values of applicants to sports physical therapy residency and fellowship programs and contrast these perspectives with the perceptions of the faculty members who provide these training experiences. The survey respondents appear to be representative of the subjects of interest and their demographic characteristics are representative of the AASPT membership and other research projects of similar intent. ${ }^{4-8,14,15,17}$

The variables that influence a decision to pursue sports physical therapy residency and specialization credential have remained relatively consistent over the past 20 plus years. ${ }^{16,17}$ It appears that applicant's impetus to pursue residency training is intrinsically driven as they demonstrate autonomous motivations that fully endorse and show commitment to training for the sake of training. They pursue residency opportunities to access accomplished mentors who will enhance their abilities to think, reason, and appropriately apply evidence in their decision-making in a 
specific discipline in which they can establish a professional network of colleagues. The applicants appear to value personal growth, achievement, and knowledge acquisition that will benefit both their athletic patients and the profession in which they serve. They are less inspired by externally controlled rewards such as titles, recognition from other health care providers, or the capacity for improving their future salary compensation package. These factors are very consistent with the findings of Gusman et al and Osborne et al from broader surveys of physical therapy residency applicants. ${ }^{9,10}$

Although only ranked at the "very important" level, residency/fellowship applicants rated the development of their future leadership skills, the establishment of life-long learning habits, and contributions to the evolution of sports physical therapy at a significantly higher level of importance than was perceived by program faculty. This finding offers further evidence to endorse the intrinsic behavioral nature that stimulates interest and commitment to residency or fellowship training in these post-professional learners. ${ }^{9,10,21}$

The results of this survey offer keen insight to program directors, coordinators, and faculty as to what specific types of training opportunities are valued by applicants. While the applicants do not seem to be as concerned by the level of salary compensation they do seem to visualize residency training programs as a means to identifying and finding future job opportunities in the field. The job opportunities specific to sports physical therapy, particularly at the highest levels of competition (collegiate and professional environments), are limited and it appears that training applicants view advanced training as a means to be familiar with the landscape, access the influential decision-makers, and acquire the skills necessary to be viable in this relatively narrow field of job opportunities. This factor was the only motivation rated as "extremely important" by the applicant cohort. This finding is consistent with the finding of Briggs et al that identified that employers rate residency and fellowship-trained clinicians superior in the domains of leadership, communication, clinical aptitude, scholarship, and teaching. ${ }^{14}$ Residency faculty should be cognizant of this variable and ensure that the training circumstance they provide is consistent with the applicant's future employment aspirations.

Other incentives that were rated significantly higher by applicants as a rationale for applying to a particular residency program centered on educational opportunities. Resident applicants highly valued a residency program housed within an academic institution with ample opportunity for supplemental learning activities and the chance to teach. It is unclear if this tendency was influenced by the nature of the survey items or if the invitations to potential applicants were biased by a larger percentage of academic education providers encouraging participation. However, this sentiment is consistent with the findings of Hartley et al in their survey of applicants from a variety of specialty disciplines in physical therapy. ${ }^{11}$

Motivations that may have been overrated by program faculty as a rationale for application to a particular program included the perceived reputation of the program, the program's past specialty examination pass rate, the geographi- cal location of the program, and the needs/desires of the applicant's significant other. The considerations for location and needs of a significant other may be mitigated by the fact that the typical residency training commitment is only for one year and many applicants and their spouses may be young enough to not yet have established familial or occupational roots in a particular community. While still rated as "very important" by applicants, residency faculty may be surprised by the comparatively lower significance assigned to program reputations and exam pass rates. The extremely high historical pass rates for all residency program graduates and high accreditation benchmarks may assure applicants that all programs have high standards and successful examination outcomes.

Analysis of the results highlighted other areas that both cohorts concur to be of higher importance in identifying desirable training opportunities. These include the recognition of a kindred connection with program faculty who possess exemplary qualifications and experience. Additionally, it appears that the applicant's personal network of advice from respected mentors and colleagues is valued more than the general reputation of a particular program. Variables that seem to have little influence on a training site's appeal include the availability of part-time participation, the number of other residents in the training cohort, the ability to moonlight during residency, or the future educational training opportunities at the residency institution.

To make intelligent decisions on where to apply or accept post-professional training opportunities it is necessary to acquire, exchange, and disseminate information between the training sites and the potential applicant candidates. The survey results indicate that both cohorts have similar perspectives on the most effective ways to communicate program information. Both groups valued email and phone contact with program personnel as influential in deciding where to apply and using the interview day to clarify how well the needs of both entities could be met. The face-toface interaction, typically offered on an interview day, was rated as extremely important in helping each party decide upon the suitability of the applicant and the congruency of the desired learning opportunity. The survey did not evaluate the benefit or impact of the Mobilize platform provided by the AASPT web site to help inform applicants of the unique characteristics inherent to each residency training program as it was not available at the time of the investigation. It is likely this vehicle will become a valuable repository of residency program information that will be beneficial to all sports physical therapy academy members.

The final section of the survey evaluated which factors and criteria are influential in matching residents to programs. In all but one instance, the resident and faculty cohorts agreed on the importance of each potential selection criterion. The variables that were rated by both groups as "extremely important" could be divided into factors that helped the applicant get an interview and the criterion that was used to distinguish which of those interviewed were offered residency employment. Letters of recommendation from applicant's faculty, clinical instructors, and previous employers along with the content of their essay question responses were highly rated as a means to identify applicants that could be successful in a given program. The ap- 
plicant interview performance, previous relationships with program faculty, and personal certifications, licenses, and experience all were important in honing the application field down to those who receive an appointment offer. The one item in which residents perceived the program would rate as more important was the likelihood of the resident staying with the institution after the conclusion of their training. This finding would indicate that programs do not necessarily view residency training programs as an employee retention tool although the nature of this study design cannot be conclusive in this perspective.

Despite an array of noteworthy findings, this descriptive study is not without limitations. While the survey appears to be comprehensive in scope it is possible that influential characteristics, factors, or criteria were not evaluated. Additionally, the applicant cohort included all repsondents who indicated they had applied to a residency program independent of acceptance or completion of the program. Similarly, the program cohort represented both program directors and faculty. In both cases, no attempt was made to distinguish the perspectives of the different types of survey respondents assigned to each group. Also, the survey did not identify the type of sponsoring programs (hospitalbased, academic, private-practice, etc) so it is not possible to generalize these findings to a specific type of organizational structure. Additionally, the nature of the survey did not allow the respondents to request clarifications on survey questions which allows for the possibility of some items being erroneously interpreted by the respondent. While the response rate of $69 \%$ is high, it does not represent all programs and has a 5\% margin of error. It is also important to note that these results only reflect the perspectives of personnel involved with sports physical therapy post-professional training. Consequently, the results of this survey should not be generalized to other specialty disciplines accredited by the ABPTRFE. As the purpose of the project was exploratory, it should be noted that Bonferonni correction for multiple comparisons were not conducted so there is a likelihood that many, if not all, of the factors may not represent significant differences between applicant and faculty cohorts.

\section{CONCLUSION}

Post-professional residency and fellowship training appears to be a relationship-focused interaction. Both faculty and applicants value direct communication and acknowledge the importance and worth of mentorship-based communications and the establishment of long term network relationships. Sports resident applicants are particularly motivated by the opportunity to make connections in a niche field of practice and perceive residency and fellowship training as means by which to enhance their employability in a competitive job market.

While the motivations for residency training may be slightly different between residency provider and recipient cohorts the importance of information acquisition and methods for residency selection criteria seem quite congruent. However, residency faculty may underestimate the importance of some of the most important motivations that prompt interest in residency training. Chief among these motivations is the intrinsic catalyst for learning. Recognition of these factors may affect how residency program content and experiences are constructed and delivered.

\section{ACKNOWLEDGMENTS}

The author would like to acknowledge the efforts of Nick Mahaffey, PT, DPT, SCS, CSCS, Marcos Lopez, PT, DPT, OCS, and Tim Schuckers, PT, DPT, OCS in the development of the survey tool.

\section{CONFLICTS OF INTEREST}

I declare that I do not have any conflicts of interest in the authorship or publication of this contribution.

Submitted: February 01, 2021 CDT, Accepted: April 09, 2021

CDT 


\section{REFERENCES}

1. Furze JA, Tichenor CJ, Fisher BE, Jensen GM, Rapport MJ. Physical therapy residency and fellowship education: Reflections on the past, present, and future. Phys Ther. 2016;96(7):949-960. $\underline{\mathrm{d}}$ oi:10.2522/ptj.20150473

2. Hartley GW, Rapport MJ, Osborne R, Briggs MS, Jensen GM. Residency education: Is it now or never? Phys Ther. 2020;101(4). doi:10.1093/ptj/pzaa225

3. Robertson EK, Tichenor CJ. Postprofessional cartography in physical therapy: charting a pathway for residency and fellowship training. J Orthop Sports Phys Ther. 2015;45(2):57-60. doi:10.2519/jospt.2015.0 $\underline{102}$

4. Mulligan EP, Rauh M, Heiderscheit B, et al. Sports physical therapy education in the United States: Where do we go from here? J Allied Health. 2020;49(2):e79-e87.

5. Mulligan EP, DeVahl J. Sports physical therapy curricula in physical therapist professional degree programs. Int J Sports Phys Ther. 2017;12(5):787-797. doi:10.26603/ijspt20170787

6. Mulligan EP, Weber MD, Reinking MF. Competency revalidation study of specialty practice in sports physical therapy. Int J Sports Phys Ther. 2014;9(7):959-973.

7. American Physical Therapy Association. American Board of Physical Therapy Residency and Fellowship Education: Description of Residency Practice. htt p://www.abptrfe.org/uploadedFiles/ABPTRFEorg/Fo r Programs/Apply/Forms/DRP Sports.pdf. Accessed January 24, 2021.

8. American Board of Physical Therapy Residency and Fellowship Education. http://www.abptrfe.org/Hom e.aspx. Accessed January 24, 2021.

9. Osborne R, Janson C, Black L, et al. Motivations to pursue physical therapy residency training: A QMethodology study of stakeholder perspectives. Phys Ther. 2020;100(1):57-72.

10. Gusman LN, Hartley G, Harrington KL, et al. Factors influencing the decision to apply to physical therapist residency program. Poster presented at APTA Combined Sections Meeting. 2020 Feb 12-15; Denver, CO.
11. Hartley G, Harrington KL, Gusman LN, et al. Program characteristics influencing application to a physical therapist residency program. Poster presented at APTA Combined Sections Meeting. 2020 Feb 12-15; Denver, CO.

12. Dickson T, Mulligan EP, DeVahl J. The toll of student debt: Financial stress and post-professional career selection among health profession students. $J$ Phys Ther Educ. 2020;34(4):339-346. doi:10.1097/jte.0 000000000000162

13. American Academy of Sports Physical Therapy. Specialization Special Interst Group. https://aaspt.me mberclicks.net/specialization-sig. Accessed January 24, 2021.

14. Briggs MS, Whitman J, Olson-Kellogg B, et al. Employer perceptions of physical therapists' residency and fellowship training: Insights for career development planning. J Phys Ther Educ. 2019;33(1):40-48. doi:10.1097/ite.0000000000000078

15. Chapman M, Lehman E, Pedersen L, et al. Best practices for occupational therapy fellowship and physical therapy residency programs: A mixed model study. Internet J of Allied Health Sciences and Practice. 2019;17(4):1540-1548.

16. Smith KL, Tichenor CJ, Schroeder M. Orthopaedic residency training: a survey of gradutes' perspectives. J Orthop Sports Phys Ther. 1999;29(11):635-651. doi:1 $\underline{0.2519 / \text { iospt.1999.29.11.635 }}$

17. Mulligan EP. Attributes, attitudes, and specialization examination success of residencytrained sports physical therapists. Poster presented at Texas Physical Therapy Association Annual Conference. 2014 October; Galveston, TX.

18. Harris PA, Taylor R, Thielke R, Payne J, Gonzalez $\mathrm{N}$, Conde JG. Research electronic data capture (REDCap) - A metadata-driven methodology and workflow process for providing translational research informatics support. J Biomed Inform. 2009;42(2):377-381. doi:10.1016/i.jbi.2008.08.010

19. Arena R, Girolami G, Aruin A, Keil A, Sainsbury J, Phillips SA. Integrated approaches to physical therapy education: A new comprehensive model from the University of Illinois Chicago. Physiother Theory Pract. 2017;33(5):353-360. doi:10.1080/09593985.201 7.1305471

20. Concepts and Application of Interferential Statistics. http://www.vassarstats.net/. Accessed January 24, 2021. 
21. Ryan RM, Deci EL. Self-determinatoin theory and the facilitation of intrinsic motivation, social development, and well-being. Am Pyschol. 2000;55(1):67-78. 